\title{
GAUSSIAN CURVATURES OF LORENTZIAN METRICS ON THE PLANE AND PUNCTURED PLANES
}

\author{
JIANGFAN LI \\ (Communicated by Jonathan M. Rosenberg) \\ Dedicated to Professors Gu Chaohao and Hu Hesheng
}

\begin{abstract}
We prove that every $f \in C^{k}\left(R^{2}\right)$ is the Gaussian curvature of some $C^{k+1}$-Lorentzian metric $(0 \leq k \leq \infty)$. Let $M$ denote the cylinder. We prove that every continuous function on $M$ is the Gaussian curvature of some $C^{1}$-Lorentzian metric. If $f \in C^{k}(M)$ satisfies the condition (H) in the Lemma 2 below, then it is the curvature function of some $C^{k+1}$-Lorentzian metric. If $f \in C^{k}\left(R^{2}\right) \quad(1 \leq k \leq \infty)$ has compact support, then the Lorentzian metric can be made complete.
\end{abstract}

\section{INTRODUCTION}

Given a function on the surface, does there exist a metric whose Gaussian curvature is the given function? This is to solve the second order nonlinear partial differential equation:

$$
K(l)=f,
$$

where $f$ is the given function, $K(l)$ is the Gaussian curvature of the definite or indefinite metric $l$. Many mathematicians have studied the case when $l$ is a Riemannian metric. For the Lorentzian metric $l$, Burns [1] got some results in 1977. In this note we are going to solve (1) on the 2-dimensional plane and to make a try on punctured planes (skirts, shirts, T-shirts, etc.).

\section{CuRvature functions on the Plane}

Lemma 1. Suppose $h \in L^{1}\left(R^{2}\right),\|h\|_{L^{1}\left(\mathbf{R}^{2}\right)}<1 / \pi$; then

$$
w(x, y)=\int_{0}^{x} \int_{0}^{y} h(s, t) e^{w(s, t)} d s d t \quad \text { on } R^{2}
$$

admits a unique solution $w \in C\left(R^{2}\right)$.

Received by the editors September 14, 1988 and, in revised form, January 23, 1989.

1980 Mathematics Subject Classification (1985 Revision). Primary 53C50, 53B30; Secondary $35 \mathrm{~L} 10$.

Key words and phrases. Curvature function, (complete) Lorentzian metric, characteristic line, (forward) complete geodesic.

The research was supported by a University of Pennsylvania Graduate Fellowship.

(C) 1990 American Mathematical Society $0002-9939 / 90 \$ 1.00+\$ .25$ per page 
Proof. Define a sequence by

$$
\begin{aligned}
u_{0} & =0, \\
u_{n+1}(x, y) & =\int_{0}^{x} \int_{0}^{y} h(s, t) e^{u_{n}(s, t)} d s d t, \quad n=0,1,2, \ldots ;
\end{aligned}
$$

then $\left\|u_{n}\right\|_{C\left(R^{2}\right)}<1,\left\|u_{n+1}-u_{n}\right\|_{C\left(R^{2}\right)}<(e / \pi)\left\|u_{n}-u_{n-1}\right\|_{C\left(R^{2}\right)}$. Hence $\left\{u_{n}\right\}$ converges uniformly on $R^{2}$ and the limit function is a solution of the equation (2). The uniqueness is obvious. Q.E.D.

Theorem 1. Let $k$ be a nonnegative integer or $k=\infty$. Suppose $f \in C^{k}\left(R^{2}\right)$. Then there exists a $C^{k+1}$-Lorentzian metric on $R^{2}$ which is pointwise conformal to the standard flat Lorentzian metric $d x d y$ such that its Gaussian curvature equals $f$.

Proof. Let $G \in C^{\infty}(R)$ such that, for all $t \in R$

$$
G(t)>\max \{\pi(|f(x, y)|+1)|| x|\leq| y|=| t \mid \text { or }|y| \leq|x|=|t|\} .
$$

Let

$$
h(x, y)=-\frac{1}{2} \frac{f(x, y)}{G(x) G(y)} e^{-x^{2}-y^{2}}
$$

then

$$
\|h\|_{L^{1}\left(R^{2}\right)}<\frac{1}{\pi} .
$$

By Lemma 1, there exists $w \in C\left(R^{2}\right)$ satisfying the equation (2). Then

$$
u(x, y)=w(x, y)-x^{2}-y^{2}-\ln (G(x) G(y))
$$

satisfies

$$
u_{x y}=-\frac{1}{2} f(x, y) e^{u} \quad \text { on } R^{2},
$$

which means the metric $e^{u} d x d y$ has the Gaussian curvature $f$. Q.E.D.

As a consequence, given a $C^{k}$-function $f$ on any 2-manifold $M$, for each point $p \in M$, locally there always exists a $C^{k+1}$-Lorentzian metric whose Gaussian curvature equals $f$ in a neighborhood of $p$.

\section{CURvature functions ON A PUNCTURED Plane}

Lemma 2. Let $k$ be a nonnegative integer or $k=\infty, p=\left(x_{0}, y_{0}\right) \in R^{2}$. Suppose $f \in C^{k}\left(R^{2} \backslash\{p\}\right)$ satisfies the following condition:

For each $n(0 \leq n<k+1)$, there exists a function $F \in$ (H) $\quad L^{1}[-1,1]($ set $F(0)=+\infty)$, such that, for $(x, y) \in[-1,1]^{2} \backslash$ $\{(0,0)\}$,

$$
\left|\frac{\partial}{\partial x^{n}} f\left(x+x_{0}, y+y_{0}\right)\right|+\left|\frac{\partial}{\partial y^{n}} f\left(x+x_{0}, y+y_{0}\right)\right|<\min \{F(x), F(y)\} .
$$


Then any solution of the equation (2) (replacing $h$ by $f$ ) is in fact in $C^{k+1}\left(R^{2} \backslash\{p\}\right)$.

Proof. By Lebesgue's dominated convergence theorem. Q.E.D.

Theorem 2. Let $k$ be a nonnegative integer or $k=\infty . \quad p=(0,0) \in R^{2}$. Suppose $f \in C^{k}\left(R^{2} \backslash\{p\}\right)$ satisfies the condition $(H)$ of Lemma 2; then there exists a $C^{k+1}$-Lorentzian metric on $R^{2} \backslash\{p\}$ such that its Gaussian curvature equals $f$.

Proof. There is $G \in C^{\infty}(R)$ satisfying (3) for $|t|>1$. Then

$$
g(x, y)=-\frac{f(x, y)}{2 G(x) G(y)} e^{-x^{2}-y^{2}}
$$

is integrable on $R^{2}$. Pick $\varepsilon>0$ such that

$$
h=\varepsilon g
$$

satisfies $\|h\|_{L^{1}\left(R^{2}\right)}<1 / \pi$. Clearly $h$ also satisfies the condition $(\mathrm{H})$ of Lemma 2. Hence the solution $w$ to the equation (2) (in Lemma 1) is in $C^{k+1}\left(R^{2} \backslash\{p\}\right)$ by Lemma 2 , and so is the function $u$ which is defined by

$$
u(x, y)=w(x, y)-x^{2}-y^{2}-\ln (G(x) G(y))+\ln \varepsilon .
$$

Then the Lorentzian metric $e^{u} d x d y$, which has the Gaussian curvature $f$, is of class $C^{k+1}$. Q.E.D.

Remark 1. Let $M=R^{2} \backslash\{$ finite points $\}, f \in C^{k}(M)$. If for each $\left(x_{0}, y_{0}\right) \in$ $R^{2} \backslash M, f$ satisfies the condition $(\mathrm{H})$ of Lemma 2 , then the same conclusion holds.

Theorem 3. On a cylinder, every continuous function is the Gaussian curvature for some $C^{1}$-Lorentzian metric.

Proof. Look at a cylinder as $R^{2} \backslash\{p\}$, say, $p=(0,0)$. Suppose $f \in C\left(R^{2} \backslash\{p\}\right)$. We first shrink $f$ into control. Pick $g \in C\left(R^{2} \backslash\{(0,0)\}\right)$, such that

$$
\begin{gathered}
g(x, y)=h(r) \text { depends only on } r=\sqrt{x^{2}+y^{2}}, \\
g>f \text { on } R^{2} \backslash\{(0,0)\}, \\
h(r) \text { is decreasing for } r \in(0,2] .
\end{gathered}
$$

Let $\varphi$ be a $C^{\infty}$-diffeomorphism of $(0,+\infty)$ such that $\left.\varphi\right|_{(1,+\infty)}=$ id and $h(\varphi) \in L^{1}(0,1]$. Define

$$
\begin{gathered}
\Phi: R^{2} \backslash\{(0,0)\} \rightarrow R^{2} \backslash\{(0,0)\}, \\
\Phi(x, y)=\left(\frac{\varphi(r)}{r} x, \frac{\varphi(r)}{r} y\right),
\end{gathered}
$$

where $r=\sqrt{x^{2}+y^{2}}$. Define

$$
F(t)=h(\varphi(|t|)) \quad \text { for } t \neq 0, \quad \text { and } F(0)=+\infty \text {. }
$$


Then $F \in L^{1}[-1,1]$ and for $(x, y) \in[-1,1]^{2} \backslash\{(0,0)\}$,

$$
\begin{aligned}
f(\Phi(x, y)) & =f\left(\frac{\varphi(r)}{r} x, \frac{\varphi(r)}{r} y\right)<g\left(\frac{\varphi(r)}{r} x, \frac{\varphi(r)}{r} y\right) \\
& =h(\varphi(r))=F(r) \leq \min (F(x), F(y)) .
\end{aligned}
$$

Applying Theorem 2 we know that $f(\Phi)$ is the curvature function of some $C^{1}$ Lorentzian metric $l$. Hence the pull-back metric $\left(\Phi^{-1}\right)^{*} l$ has the curvature f. Q.E.D.

Remark 2. The same conclusion holds for the manifold $R^{2} \backslash A$ where $A$ is a discrete subset of $R^{2}$.

\section{CURVATURE FUnCTIONS FOR COMPLETE LORENTZIAN METRICS}

Throughout this section, we use the notation $B_{r}=\left\{x^{2}+y^{2} \leq r^{2}\right\}$. For the Lorentzian metric $e^{u} d x d y$ on $R^{2}$, the equations of the geodesic are

$$
\begin{gathered}
\ddot{x}+u_{x} \dot{x}^{2}=0, \\
\ddot{y}+u_{y} \dot{y}^{2}=0 .
\end{gathered}
$$

In particular, the characteristic lines (i.e. those lines parallel to the $x$-axis or $y$-axis) are geodesics. We say a geodesic $\gamma(t)$ is forward complete (complete, respectively) if it exists for all $t \geq 0$ (all $t$, resp.). Note the fact that for a positive function $f \in C(R)$, the solution to the problem

$$
\dot{x}=f(x), \quad x(0)=x_{0}
$$

exists for all $t \geq 0$ if and only if

$$
\int_{x_{0}}^{+\infty} \frac{d x}{f(x)}=+\infty
$$

This implies

Lemma 3. Let $u \in C\left(R^{2}\right)$. The characteristic lines of the metric $e^{u} d x d y$ are complete if and only if for any $x$ and $y$,

$$
\int_{0}^{\infty} e^{u(x, y)} d x=\int_{0}^{\infty} e^{u(x, y)} d y=\int_{-\infty}^{0} e^{u(x, y)} d x=\int_{-\infty}^{0} e^{u(x, y)} d y=+\infty
$$

Proof. Consider the characteristic line $\gamma(t)=\left(x(t), y_{0}\right)$. It is a geodesic if properly parametrized:

$$
\ddot{x}(t)=-u_{x}\left(x(t), y_{0}\right) \dot{x}(t)^{2}, \quad x(0)=x_{0}, \quad \dot{x}(0) \neq 0 .
$$

Let

$$
p(t)=\dot{x}(t)
$$

then

Therefore

$$
\frac{d p}{d x}=-u_{x}\left(x, y_{0}\right) p .
$$

$$
p=c e^{-u\left(x, y_{0}\right)} \quad\left(c=\dot{x}(0) e^{u\left(x_{0}, y_{0}\right)} \neq 0\right) .
$$


That is

$$
\frac{d x}{d t}=c e^{-u\left(x, y_{0}\right)} \text {. }
$$

Hence $\gamma(t)$ is forward complete if and only if $x(t)$ is, if and only if

$$
\int_{x_{0}}^{\infty} e^{u\left(x, y_{0}\right)} d x=+\infty \quad(\text { if } c>0)
$$

or

$$
\int_{-\infty}^{x_{0}} e^{u\left(x, y_{0}\right)} d x=+\infty \quad(\text { if } c<0) .
$$

All characteristic lines are complete if and only if every characteristic line is forward complete, if and only if (4) holds. Q.E.D.

Remark 3. By Lemma 3 one can see that the metric constructed in the proof of Theorem 1 is not complete.

Corollary 1. If $u \in C\left(R^{2}\right)$ satisfies

$$
\liminf _{r \rightarrow \infty R \geq r}\left(\|u\|_{C\left(B_{R}\right)}-\ln R\right)<+\infty,
$$

then every characteristic line of the Lorentzian metric $e^{u} d x d y$ on $R^{2}$ is complete.

Proof. It is equivalent to prove that the integrals in Lemma 3 are $+\infty$. From the condition, there exist a sequence $r_{n} \rightarrow+\infty$ and a constant $c>0$ such that

$$
\|u\|_{C\left(B_{r_{n}}\right)}-\ln r_{n} \leq \ln c \text {. }
$$

Then

$$
e^{u} \geq \frac{1}{c r_{n}} \quad \text { on } B_{r_{n}}
$$

Fix $y$. Define

$$
x_{n}=\sqrt{r_{n}^{2}-y^{2}}
$$

By passing to a subsequence we may assume all $r_{n}>|y|$ and

$$
\frac{x_{n}-x_{n-1}}{r_{n}}>\frac{1}{2} \quad \text { for all } n \text {. }
$$

Then

$$
\begin{aligned}
\int_{0}^{\infty} e^{u(x, y)} d x & \geq \sum_{n} \int_{x_{n-1}}^{x_{n}} e^{u(x, y)} d x \\
& \geq \sum_{n} \frac{1}{c} \frac{x_{n}-x_{n-1}}{r_{n}} \\
& =+\infty \quad(\text { by }(6)) .
\end{aligned}
$$

Similarly the other three integrals also equal $+\infty$. Then Lemma 3 applies. Q.E.D. 
Theorem 4. If $u \in C^{1, \alpha}\left(R^{2}\right) \quad(0<\alpha<1)$ satisfies

$$
\liminf _{r \rightarrow \infty R \geq r}\left(\|u\|_{C^{1}\left(B_{R}\right)}-\frac{1}{2} \ln \ln R\right)=-\infty
$$

then $e^{u} d x d y$ is a complete Lorentzian metric on $R^{2}$.

Proof. By Corollary 1, every characteristic line is complete. Now consider an arbitrary noncharacteristic geodesic $\gamma(t)=(x(t), y(t))(\dot{x}(t) \dot{y}(t) \neq 0)$. It suffices to prove that $\gamma$ is forward complete. Define the notations

$$
\begin{gathered}
p(t)=\dot{x}(t), q(t)=\dot{y}(t), \\
\alpha(t)=\frac{1}{p(t)}, \beta(t)=\frac{1}{q(t)}, \\
X(t)=(x(t), y(t), \alpha(t), \beta(t)), \\
\|(x, y, z, t)\|=\sqrt{x^{2}+y^{2}+z^{2}+t^{2}}, \\
\|X\|_{C[0, T]}=\sup _{t \in[0, T]}\|X(t)\| .
\end{gathered}
$$

The condition implies that there exist sequences

$$
r_{n} \rightarrow+\infty \text { and } c_{n} \rightarrow+\infty
$$

such that for all $n$,

$$
\|u\|_{C^{1}\left(B_{r_{n}}\right)} \leq \frac{1}{2} \ln \ln r_{n}-c_{n} .
$$

We may assume $\left\{\frac{1}{2} \ln \ln r_{n}-c_{n}\right\}$ is an increasing sequence. Pick an increasing function $c(r)$ such that, for all $r \geq 0$,

$$
\|u\|_{C^{1}\left(B_{r}\right)} \leq c(r)
$$

and for all $n$,

$$
c\left(r_{n}\right)=\frac{1}{2} \ln \ln r_{n}-c_{n} .
$$

For an arbitrary $L>0$, we are going to estimate

$$
T(L)=\sup \left\{t \mid X([0, t]) \text { exists and }\|X\|_{C[0, t]} \leq L\right\} .
$$

Note that along a geodesic $\gamma(t), e^{u(\gamma(t))} p(t) q(t)=$ constant. Hence

$$
p(t) q(t)=e^{u(\gamma(0))-u(\gamma(t))} p(0) q(0) .
$$

Denote

$$
a(L)=e^{2 c(L)}|p(0) q(0)| .
$$

Then (9) and (11) show that for $t \in[0, T(L)]$,

$$
\begin{gathered}
|p(t) q(t)| \leq a(L), \\
p^{2}(t)+q^{2}(t)=p^{2} q^{2}\left(\alpha^{2}+\beta^{2}\right) \leq a(L)^{2}\|X(t)\|^{2}, \\
\left|u_{x}(\gamma(t))\right|+\left|u_{y}(\gamma(t))\right| \leq 2 c(L) .
\end{gathered}
$$


Since $\gamma$ is the geodesic of $e^{u} d x d y$, therefore

$$
X(t)=X_{0}+\int_{0}^{t}\left(p(t), q(t), u_{x}(\gamma(t)), u_{y}(\gamma(t))\right) d t,
$$

where $X_{0}=(x(0), y(0), \alpha(0), \beta(0))$ is the initial data. From (13)-(15), we obtain that, for $t \in[0, T(L)]$,

$$
\|X(t)\| \leq\left\|X_{0}\right\|+\int_{0}^{t}(2 c(L)+a(L)\|X(t)\|) d t .
$$

Let

$$
f(t)=\int_{0}^{t}\|X(t)\| d t
$$

then (16) is

$$
\begin{aligned}
f^{\prime}(t) & \leq\left\|X_{0}\right\|+2 c t+a f(t), \\
\frac{d}{d t}\left(e^{-a t} f(t)\right) & =e^{-a t}\left(f^{\prime}-a f\right) \leq e^{-a t}\left(\left\|X_{0}\right\|+2 c t\right),
\end{aligned}
$$

where $a=a(L)$ and $c=c(L)$. Integrating both sides, we get

$$
\begin{aligned}
e^{-a t} f(t) & \leq \frac{2 c}{a^{2}}+\frac{\left\|X_{0}\right\|}{a}-e^{-a t}\left(\frac{2 c}{a} t+\frac{2 c}{a^{2}}+\frac{\left\|X_{0}\right\|}{a}\right), \\
f(t) & \leq\left(\frac{2 c}{a^{2}}+\frac{\left\|X_{0}\right\|}{a}\right)\left(e^{a t}-1\right)-\frac{2 c}{a} t .
\end{aligned}
$$

Then (16)-(18) imply that, for $t \in[0, T(L)]$,

$$
\|X(t)\| \leq M_{L}(t)
$$

where $M_{L} \in C^{\infty}(R)$ is defined as

$$
M_{L}(t)=\left\|X_{0}\right\|+\left(\frac{2 c(L)}{a(L)}+\left\|X_{0}\right\|\right)\left(e^{a(L) t}-1\right) .
$$

Define

$$
t_{n}=\frac{1}{a\left(r_{n}\right)} \ln \frac{a\left(r_{n}\right) r_{n}+2 c\left(r_{n}\right)}{a\left(r_{n}\right)\left\|X_{0}\right\|+2 c\left(r_{n}\right)}
$$

that is

$$
M_{r_{n}}\left(t_{n}\right)=r_{n}
$$

Then (19) shows that, as long as $X(t)$ exists,

$$
\|X(t)\| \leq r_{n} \quad \text { for } t \in\left[0, t_{n}\right] .
$$

But (8),(10), (12) and (20) imply

$$
t_{n} \rightarrow+\infty \quad(n \rightarrow \infty) .
$$

Then (21) and (22) imply that there exists $L \in C(R)$ such that for all $t \geq 0$, as long as $X(t)$ exists,

$$
\|X(t)\| \leq L(t)
$$


Now (13) and (23) imply a priori estimate

$$
x(t)^{2}+y(t)^{2}+p(t)^{2}+q(t)^{2} \leq\left(1+a(L(t))^{2}\right) L(t)^{2},
$$

which guarantees that $\gamma(t)$ exists for all $t \geq 0$. Q.E.D.

Remark 4. If $u \in C^{1}\left(R^{2}\right)$ satisfies (7) and has the following property:

For any compact subset $K \subset R^{2}$, there exists $\varepsilon>0$ such that for any initial data $p \in K, v \in S^{1}=\left\{x^{2}+y^{2}=1\right\} \subset R^{2}$, the geodesic $\gamma(t)$ with $\gamma(0)=p$ and $\dot{\gamma}(0)=v$ exists unique on $t \in[0, \varepsilon]$;

then the Lorentzian metric $e^{u} d x d y$ on $R^{2}$ is complete. By Peano's existence theorem in the theory of ordinary differential equations, the local existence of the geodesic is always true. But the uniqueness might be false. If $u \in C^{1, \alpha}\left(R^{2}\right)$ $(0<\alpha<1)$, then the uniqueness is also true.

Theorem 5. Let $f \in C^{k}\left(R^{2}\right) \quad(1 \leq k \leq \infty)$,

$$
F(r)=\sup _{(x, y) \in B_{r}} \int_{-r}^{r}(|f(x, t)|+|f(t, y)|) d t .
$$

If there exist two nonnegative functions $g, h \in C(R)$ and a constant $c>0$ such that

$$
\|p\|_{L^{1}\left(R^{2}\right)}<\infty \quad \text { where } p(x, y)=f(x, y) e^{-g(x)-h(y)},
$$

and

$$
\liminf _{r \rightarrow \infty R \geq r}\left(c F(R)+\|g\|_{C^{1}[-R, R]}+\|h\|_{C^{1}[-R, R]}-\frac{1}{2} \ln \ln R\right)=-\infty,
$$

then $f$ is the curvature function for some complete $C^{k+1}$-Lorentzian metric $e^{u} d x d y$ on $R^{2}$.

Proof. Pick $\varepsilon>0$, such that

$$
\|\varepsilon p\|_{L^{1}\left(R^{2}\right)}<\frac{1}{\pi}
$$

and

$$
\liminf _{r \rightarrow \infty R \geq r}\left(\varepsilon e F(R)+\|g\|_{C^{1}[-R, R]}+\|h\|_{C^{1}[-R, R]}-\frac{1}{2} \ln \ln R\right)=-\infty .
$$

By Lemma 1, there exists $w \in C^{k+1}\left(R^{2}\right)$ satisfying

$$
w(x, y)=-\int_{0}^{x} \int_{0}^{y} \varepsilon p(s, t) e^{w(s, t)} d s d t .
$$

Clearly $\|w\|_{C\left(R^{2}\right)} \leq 1$. Then (25) implies, for all $r>0$,

$$
\|w\|_{C^{1}\left(B_{r}\right)} \leq 1+\varepsilon e F(r) .
$$

Let

$$
u(x, y)=w(x, y)-g(x)-h(y) .
$$


Then

$$
\|u\|_{C^{1}\left(B_{r}\right)} \leq 1+\|g\|_{C^{1}[-r, r]}+\|h\|_{C^{1}[-r, r]}+\varepsilon e F(r) .
$$

Then (24), (27) and theorem 4 imply that $e^{u} d x d y$ is a complete Lorentzian metric. (25) and (26) imply that $K\left(e^{u} d x d y\right)=2 \varepsilon f$. Then the complete metric $2 \varepsilon e^{u} d x d y$ has the curvature $f$. Q.E.D.

Remark 5. Theorem 5 also holds for $f \in C^{\alpha}\left(R^{2}\right) \quad(0<\alpha<1)$.

Taking $g=h=0$, we obtain the following:

Corollary 2. Let $f \in C^{k}\left(R^{2}\right) \quad(1 \leq k \leq \infty)$. If $\|f\|_{L^{1}\left(R^{2}\right)}<\infty$ and if there exists a constant $c$ such that

$$
\liminf _{r \rightarrow \infty R \geq r}\left(\sup _{(x, y) \in B_{R}} \int_{-R}^{R}(|f(x, t)|+|f(t, y)|) d t-c \ln \ln R\right)=-\infty,
$$

then $f$ is the curvature function for some complete $C^{k+1}$-Lorentzian metric $e^{u} d x d y$ on $R^{2}$.

\section{ACKNOWLEDGMENT}

The author thanks Professor Jerry Kazdan cordially for informing him of the reference [1]. He also thanks the referee for some suggestions.

\section{REFERENCES}

1. J. T. Burns, Curvature functions on Lorentz 2-manifolds, Pacific J. of Math. 70 (1977), 325335.

Department of Mathematics, University of Pennsylvania, Philadelphia, PenNSylVania 19104 